\title{
Chemical Vapor Deposition Growth of Large Single-crystal Bernal-stacked Bilayer Graphene from Ethanol
}

\author{
Xiao Chen ${ }^{1}$, Rong Xiang ${ }^{1 \star}$, Pei Zhao ${ }^{2}$, Hua An ${ }^{1}$, Taiki Inoue ${ }^{1}$, Shohei Chiashi ${ }^{1}$, \\ Shigeo Maruyama ${ }^{1,3 *}$ \\ 1 Department of Mechanical Engineering, \\ The University of Tokyo, 7-3-1 Hongo, Bunkyo-ku, Tokyo, 113-8656, Japan \\ 2 Institute of Applied Mechanics, \\ Zhejiang University, 38 Zheda Rd, Hangzhou, 310027, China \\ ${ }^{3}$ Energy NanoEngineering Laboratory,
}

National Institute of Advanced Industrial Science and Technology (AIST), 1-2-1 Namiki, Tsukuba, 205-8564, Japan
Corresponding authors. E-mail:
maruyama@photon.t.u-tokyo.ac.jp (Shigeo Maruyama)
xiangrong@photon.t.u-tokyo.ac.jp (Rong Xiang)
TEL: +81-3-5841-6421, Fax: +81-3-5800-6983 


\begin{abstract}
Using ethanol as a precursor, single-crystal bilayer graphene domains with dimensions up to hundreds of micrometer, one of the largest reported so far, were synthesized on $\mathrm{Cu}$ foils by chemical vapor deposition (CVD). Raman spectroscopy analysis revealed that the bilayers are homogeneously AB-stacked, with very low D-band intensity. Selected area electron diffraction analysis also confirmed the bernal stacking order and the large area crystallinity. Decreasing ethanol pressure in CVD shows an unambiguous tread from self-limited single layer to a multi-layer graphene, and there exists a narrow window of ethanol pressure which is preferred for the formation of large domain bilayer graphene. This suggests the dual effect of ethanol in graphene growth and demonstrates that the formation of different layers of graphene can be controlled by carefully tuning single parameter: ethanol pressure.
\end{abstract}




\section{Introduction}

Graphene, an $\mathrm{sp}^{2}$-bonded mono-layer carbon material, has been drawing enormous attention due to its extraordinary mechanical, thermal and electrical properties. Chemical vapor deposition (CVD) provides a reliable and consistent method for the synthesis of large-scale single-layer graphene (SLG) [1-5]. In the past few years, many efforts have been focused on enlarging the sizes of single-crystal graphene domains [6-12], in order to avoid the drawbacks brought by the existence of grain boundaries [13, 14]. To achieve this, researchers focused on reducing the partial pressure of the carbon source, tuning the $\mathrm{C}: \mathrm{H}$ ratio, smoothing the $\mathrm{Cu}$ surface, as well as adjusting oxygen content of Cu substrates [10-12]. As a result, single-crystal SLG of millimeter or even centimeter sizes were synthesized. Recently, we found that by using ethanol as the precursor, the growth of single-crystal on polycrystalline $\mathrm{Cu}$ substrates can be realized through a very simple procedure, and graphene single crystals as large as $5 \mathrm{~mm}$ were synthesized [15].

However, the zero band-gap in SLG makes it unsuitable for many applications such as the channels in field effect transistors, despite its high carrier mobility. Recently, several groups reported that a bandgap up to $250 \mathrm{meV}$ can be opened by an external electric field in AB-stacked bilayer graphene (BLG). [16, 17] CVD synthesis of continuous polycrystal AB-stacked BLG has been reported, but the size of single-crystal domains are very small compared with SLG. Besides, in order to break the self-limiting process of SLG on $\mathrm{Cu}$ surface, these previous works employed complicated pre-treatments or designed CVD process [18-23], such as spatially arranged Cu substrates [18, 21], percentage-engineered Cu-Ni alloy as substrates [19, 22], carefully adjusted nucleation pressure of methane [10, 20], and nonisothermal growth procedure 
with variable temperatures [23]. In our previous work, we've shown that when using ethanol as a precursor, simply by extending the CVD growth duration, the self-limiting behavior of SLG on Cu can be broken, resulting in a layer-by-layer epitaxial growth of equilibrium AB-stacked BLG [24]. However, the domain sizes in that case were a few micrometers and growth of large (e.g. over hundred micrometer) domain sized AB-stacked BLG remained challenging. Here, using an improved alcohol catalytic CVD (ACCVD), we report a successful preparation of single-crystal AB-stacked BLG with sizes up to $450 \mu \mathrm{m}$, which is so far the largest AB-stacked BLG reported. Balancing ethanol's etching and growth effect through adjusting the CVD pressure is found to be critical for the formation of large BLG flakes.

\section{Experimental methods}

Single-crystal BLG was synthesized using alcohol catalytic chemical vapor deposition (ACCVD), which is similar to the procedure used in the synthesis of single-crystal SLG [5]. A homemade thermal CVD system was employed, with a quartz tube as the reaction chamber. Commercial copper foils (Nilaco Corp., CU-113303) were cleaned using IPA, acetone, and $\mathrm{HCl}$, and heated in air on a hot plate at about $250{ }^{\circ} \mathrm{C}$ to oxidize the $\mathrm{Cu}$ surface. Afterwards, the foil was folded into a Cu pocket, loaded into the CVD chamber, and placed at the center of the furnace. Then the system was vacuumed to approximately $25 \mathrm{~Pa}$ by a mechanical pump for 10 minutes to remove air from the system. The CVD chamber was heated to the reaction temperature $\left(1065^{\circ} \mathrm{C}\right)$ with an $\mathrm{Ar}$ flow (300 sccm) under the pressure of $450 \mathrm{~Pa}$. After reaching $1065{ }^{\circ} \mathrm{C}$, the Ar flow was replaced by a $300 \mathrm{sccm}$ flow of $3 \% \mathrm{H}_{2}$ diluted in Ar (partial pressure of $\mathrm{H}_{2}$ is $10 \mathrm{~Pa}$ ), and a 0.06 sccm ethanol vapor flow was also introduced into the system to initiate the 
growth. The total pressure is kept at $450 \mathrm{~Pa}$, which is the key parameter compared with the CVD conditions for the synthesis of larger single-crystal SLG, since a small variation of the total pressure may result in a much lower yield of BLG, and this will be discussed in detail later. It would take 15-18 hours for the formation of $2^{\text {nd }}$ layer graphene with sizes of hundreds of microns, and approximately 20-22 hours for the full coverage of bilayer graphene.

The transfer of graphene is conducted using a wet-etching process with poly(methyl methacrylate) (PMMA) as the mediator, as has been explained in many previous works. For transfer to transmission electron microscopy (TEM) grids, the PMMA is removed by a 20-hour annealing in the environment of $\mathrm{H}_{2} / \mathrm{Ar}$ at $400^{\circ} \mathrm{C}$.

\section{Results and discussion}

After the growth of 16 hours using the conditions in Figure 1-a, we transfer the graphene to $\mathrm{Si} / \mathrm{SiO}_{2}$ substrate, and directly observe the $\mathrm{BLG}$ using an optical microscope, since in this way the contrast between SLG and BLG is very strong.
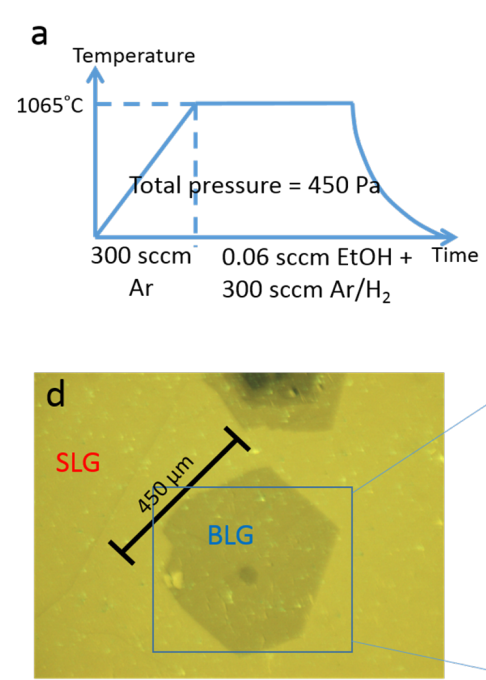
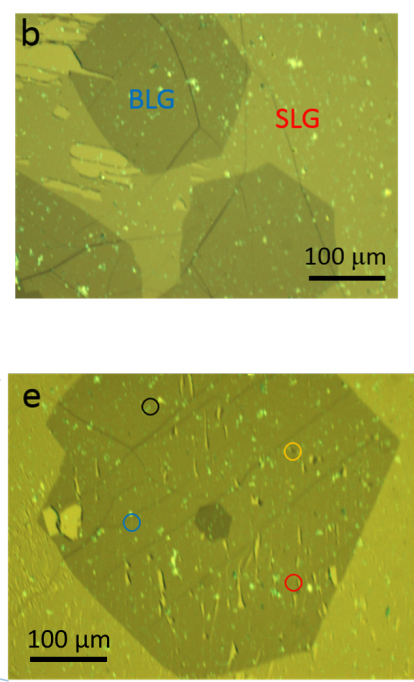
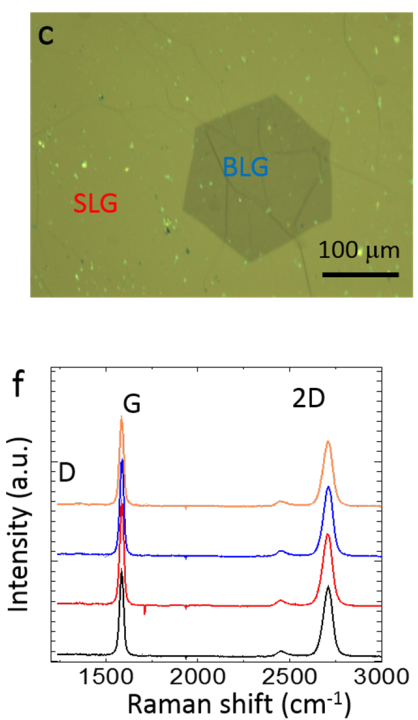
Figure 1. a) CVD conditions of the growth procedure. b) and c) Microscopic images of $\mathrm{BLG}$ on $\mathrm{Si} / \mathrm{SiO}_{2}$ substrates. d) and e) Microscopic images of a $450 \mu \mathrm{m}$ BLG hexagon. f) Raman spectra of four random locations as marked in e).

Nearly full coverage of SLG is made, and the BLG hexagons are clearly observed, as shown in Figure 1-b and c. Most of the BLG hexagons are of the edge-to-edge sizes of few tens to few hundreds of $\mu \mathrm{m}$ after an $18 \mathrm{hr}$ growth, with an average size $200 \mu \mathrm{m}$. There is a vague trend that the sizes of bilayer hexagons are larger where the nuclei are denser. This size distribution is probably caused by the unevenly distributed nucleation-preferred sites, and hence the different time needed before single-layer graphene merges in to a large polycrystal piece, since expansion of the second layer mainly occurs after the first layer merge into a continuous film. Nonetheless, more than $20 \%$ of the flakes are larger than $300 \mu \mathrm{m}$ and a $450 \mu \mathrm{m}$ (one of the largest among them) BLG region is shown in Figure 1-d.

Figure 1-f shows the Raman spectra of four randomly selected points in Figure 1-e, and they show Raman features of AB-stacked BLG, including a FWHM value of 55 $\mathrm{cm}^{-1}$, a 2D peak position at $\sim 2710 \mathrm{~cm}^{-1}$ (about $10 \mathrm{~cm}^{-1}$ up-shift compared with SLG), and that the intensity ratio of $2 \mathrm{D}$ to $\mathrm{G}$ is $\sim 1$. No obvious $\mathrm{D}$ band can be observed in these locations, indicating that the defect level is very low.

To demonstrate the homogeneity of the BLG area, we plot Raman maps of the 2D/G ratio, the full width at half maximum (FWHM) of the 2D peak, and the position of the 2D peak (Figure 2a, b, and c). In all three maps, the contrasts between the BLG area and the SLG area are very strong. Except for contaminations on the graphene film which were brought by the transfer procedure, the color on the BLG area in each map is very 
homogeneous, indicating the quality of the BLG is homogeneous. Figure 2d shows an SEM image of a BLG grown by flowing $12 \mathrm{hr}{ }^{12} \mathrm{C}$ ethanol, followed by $2 \mathrm{hr}{ }^{13} \mathrm{C}$ ethanol. Intensity plot of ${ }^{12} \mathrm{C} \mathrm{G}$ band (locating at approx. $1588 \mathrm{~cm}^{-1}$ ) and ${ }^{13} \mathrm{C} \mathrm{G}$ band (locating at approx. $1528 \mathrm{~cm}^{-1}$ ) of this sample is shown in Figure 2e, which reveals a clear enrichment of ${ }^{13} \mathrm{C}$ at the edge of the second layer in a BLG. This also confirms that the second layer in our BLG slowly grows when the first layer completely formed. Meanwhile, some red dots in Figure 2e implies that, though growth of ${ }^{13} \mathrm{C}$ mainly occurs at of the second layer, some exchange of carbon atoms in the as-grown area also happens. This may be related to the etching effect of ethanol, as to be discussed later.
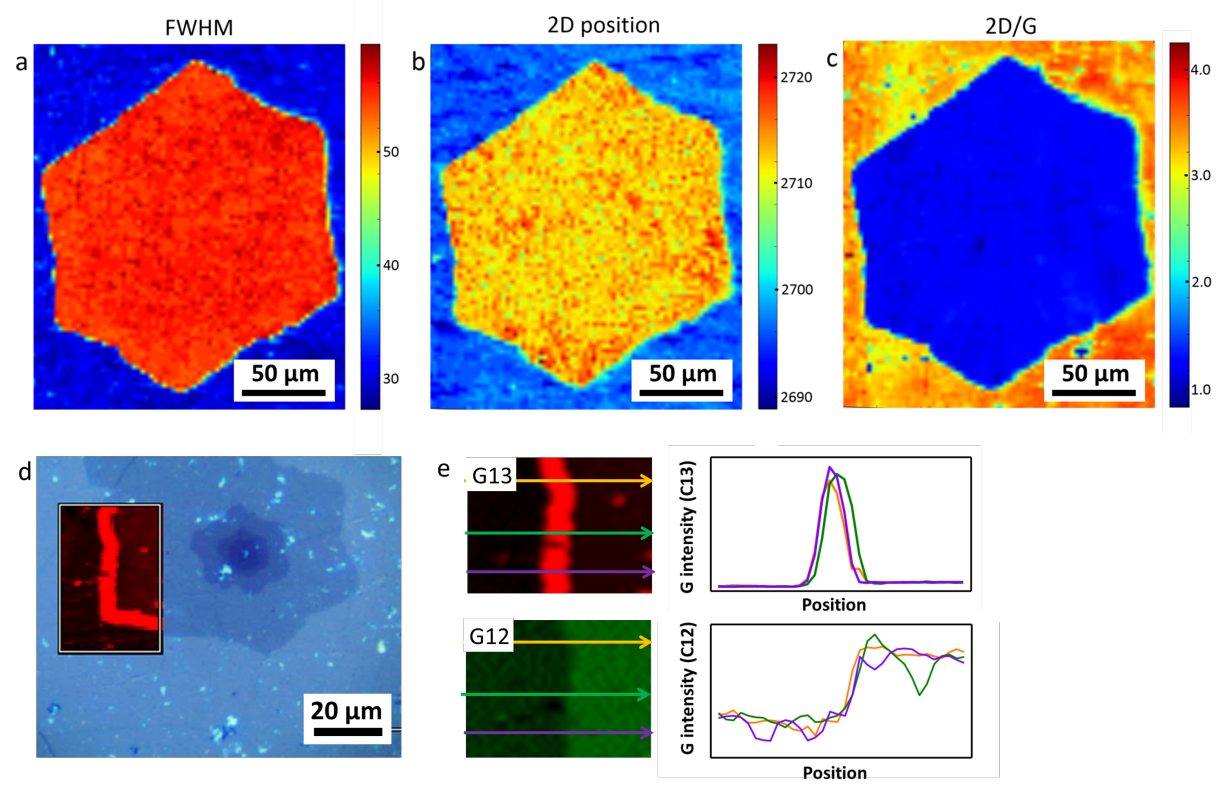

Figure 2. a) FWHM, b) $2 \mathrm{D}$ position and c) $2 \mathrm{D} / \mathrm{G}$ intensity ratio maps of a BLG hexagon; d) an SEM image a BLG grown by flowing $12 \mathrm{hr}{ }^{12} \mathrm{C}$ ethanol followed by $2 \mathrm{hr}{ }^{13} \mathrm{C}$ ethanol; e) plot of ${ }^{12} \mathrm{C}$ and ${ }^{13} \mathrm{C} \mathrm{G}$ band intensity for the sample shown in d).

Although the Raman spectra are consistent with AB-stack BLG, previous studies have shown that even twisted BLG with some certain twisted angles can exhibit Raman 
spectra that are similar to that of AB-stacked ones [25], which means that the confirmation of AB-stacked BLG only by Raman spectroscopy is not conclusive. To further confirm the stacking order and to demonstrate the single-crystal nature of the BLG hexagons, we transferred the graphene sample to a TEM grid and observed the selected area electron diffraction (SAED) patterns at different locations. The intensity of four points in Figure 3-a is shown in the inset, and the relative positions of the four locations are shown in the inset of Figure 3-b. The second-order diffractions have a stronger intensities than the first-order diffractions at all four locations, which confirms that this area is AB-stacked BLG. The angle differences of these four patterns are less than $0.8^{\circ}$, which suggests that the BLG is single-crystal at least for a distance of $200 \mu \mathrm{m}$. In most of our TEM investigations, the contrast difference between SLG and BLG is hardly distinguishable, and it is difficult to identify the number of layers from in-plane view. However, occasionally, there also exist some winkled graphene regions, which are probably caused by an imperfect transfer. These areas allow us to observe the cross-sections of graphene, and un-ambitiously confirm the enrichment of bilayer structure in our sample, as clearly shown in Figure 3 e-h. Besides BLG, SLG and few layer structure are also observed sometimes. The direct TEM imaging is fairly consistent with SEM and Raman results. 

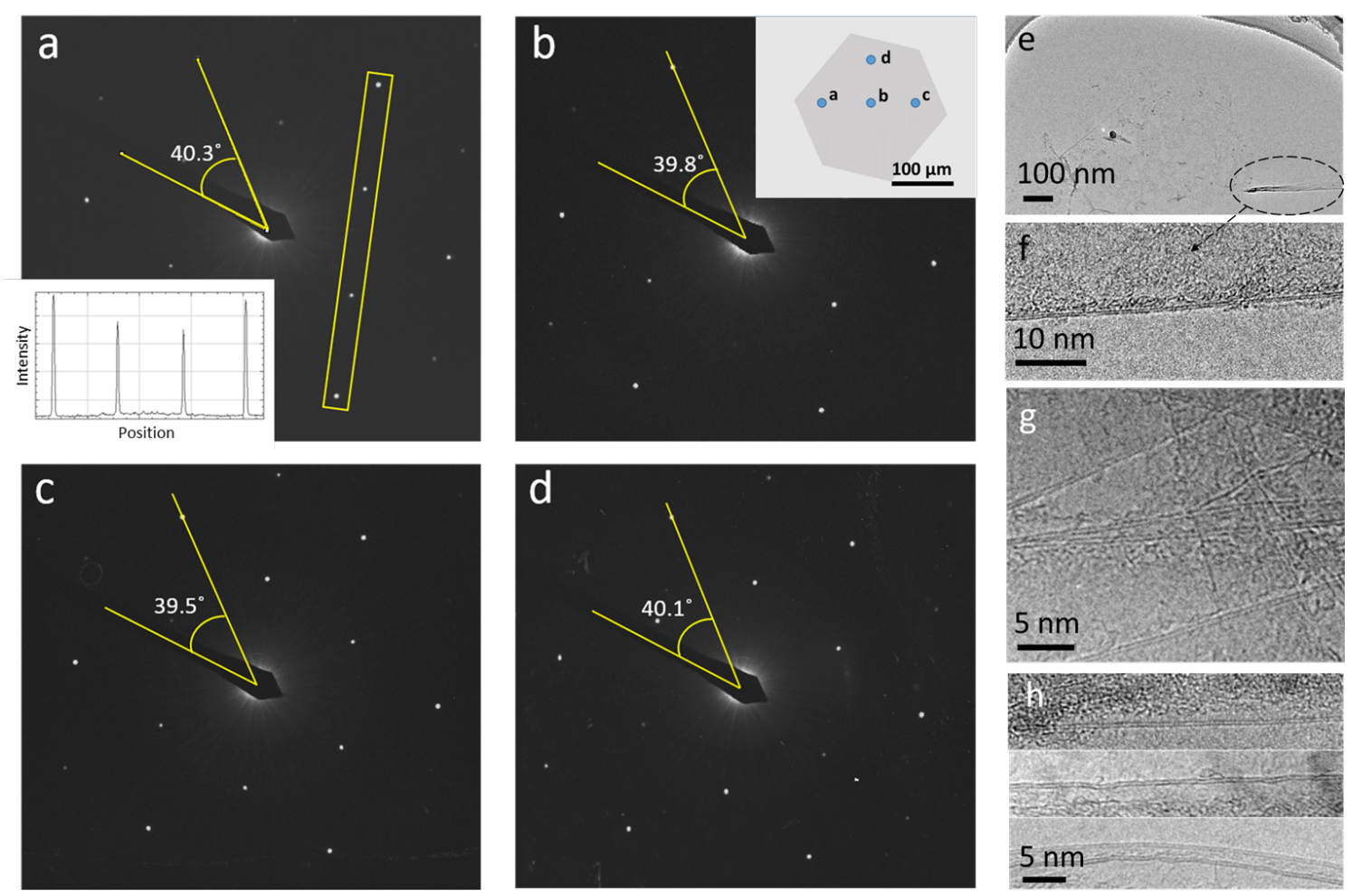

Figure 3. a) - d) SAED characterization of as-grown graphene. The relative positions are shown in the inset of b). The intensity of the four points boxed in a) is shown in the inset of a). e) - h) cross-sectional TEM images of BLG wrinkles after transfer.

Noticing that the CVD pressure plays a critical role in controlling the formation of the second layer, we investigated the growth behavior of three different pressures. When the total pressure is $300 \mathrm{~Pa}$, as has been applied in our previous work about the synthesis of large single-crystal SLG, the formation of graphene flakes was not self-limiting, i.e., the growth is not restricted to a single layer; instead, there is a multi-layer core at the center of each flake, occupying roughly 5\% of the area. Under this growth condition, after the flakes merge into a big SLG film, the nucleation and expansion of multi-layers would continue, resulting in a graphene film with many multilayer areas, as shown in Figure 4-a. However, when we raise the total pressure of 
the mixed gas in the reaction chamber during the CVD reaction, the formation of multi-layers is well refrained, and after the full coverage of SLG, only a second layer sufficiently expand, resulting in many hexagonal areas of BLG (Figure 4-b). Note that since obviously the nucleation density of the second layer is much higher than that of SLG, so many new nuclei were created during the CVD reaction other than the ones that are shared with the first layer. The pressure window for such formation of BLG is very narrow, and when we raise the pressure to $700 \mathrm{~Pa}$ (Figure 4-c), the area of BLG became very limited, leaving a majority of SLG on the $\mathrm{Cu}$ foil, and even with a longer CVD duration (24 hours), the size of BLG hexagons remains the same.

This narrow pressure window gives us an insight on the mechanism behind the successful synthesis of AB-stacked BLG. The rates of etching by the etchants (such as hydrogen and oxygen) during the CVD reaction are different depending on different layers of graphene, just as the rates of growth (carbon atoms attaching to graphene edges) are different as well. Meanwhile, the etching effect is very sensitive to pressure, while growth rate is much less dependent. At $300 \mathrm{~Pa}$, the growth rates of all layers of graphene are higher than the etching rates, hence all layers expand with extending CVD duration. At higher pressure of $450 \mathrm{~Pa}$, it is likely that the etching effect from hydrogen/oxygen increase faster than the growth rate, and for layer numbers $\geq 3$, the etching rates exceed growth rate, so only the $1^{\text {st }}$ and $2^{\text {nd }}$ layer grows. When the pressure is increased to $700 \mathrm{~Pa}$, even for the $2^{\text {nd }}$ layer, the growth rate and the etching rate reaches equilibrium, and the SLG formation on $\mathrm{Cu}$ foils becomes self-limiting (Figure 4-d). Some phenomena observed in the CVD synthesis of SLG from methane help to understand this mechanism. For instance, H. Zhou and colleagues showed that even with the same $\mathrm{H}_{2}: \mathrm{CH}_{4}$ ratio, the expansion rate of SLG decrease dramatically $(\times 1 / 3)$ 
when the total pressure of $\mathrm{H}_{2}$ and $\mathrm{CH}_{4}$ is increased $(\times 2)$, and a dramatic change in expansion rate $(\times 2)$ when only decreasing the $\mathrm{H}_{2}$ pressure $(\times 1.5)$ [10]. The difference here is that for CVD from methane, $\mathrm{H}_{2}$ is the etchant and $\mathrm{CH}_{4}$ is the source of carbon, while in ethanol CVD, ethanol may provide both etchant (such as oxygen) and carbon source. In most of cases, the growth and etch occurs at edge of graphene, however, etching and re-growth are also sometime observed in the regions of as-formed graphene (as mentioned previously in Figure 2e). This further confirms that the overall growth is an equilibrium of growth and etching, and the CVD pressure significantly changes the competition of these two processes, resulting in a control over the formed product. It may take time to further quantify the dual role of ethanol in the formation of BLG, still it is clear that the formation of BLG can be tuned through dynamic control by the CVD pressure. Finally, beside the etching effect, oxygen atoms in ethanol may have another doping effect to the final product and therefore bring interesting properties for ethanol produced graphene. These details may also need to be clarified in the future studies.

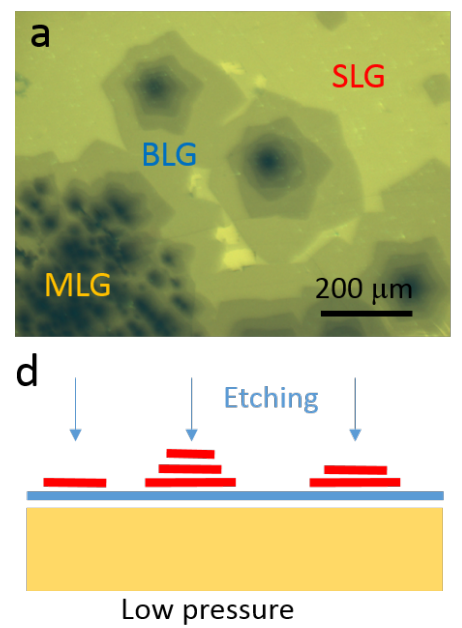

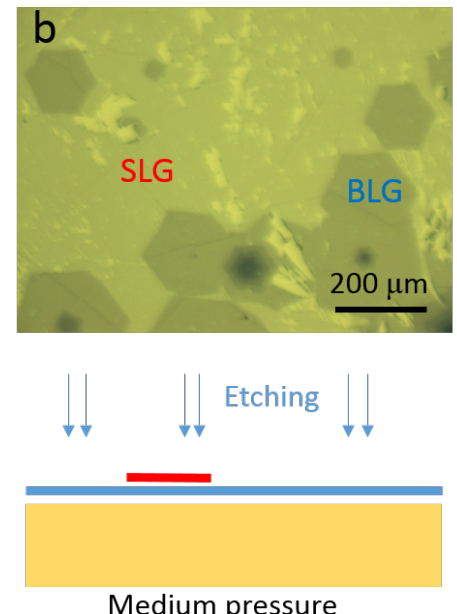

Medium pressure

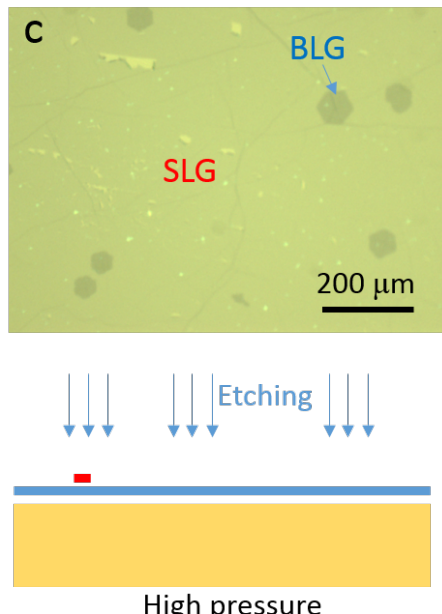

High pressure 
Figure 4. Growth pressure dependence. The three samples shown here are the result of the same CVD conditions except for the total pressure: a) $300 \mathrm{~Pa}$, b) 450 $\mathrm{Pa}$, c) $700 \mathrm{~Pa}$. The images were taken using an optical microscope after transferring graphene to $\mathrm{Si} / \mathrm{SiO}_{2}$ substrate. d) a schematic explaining the mechanism of the pressure dependence of BLG synthesis.

\section{Conclusion}

Single-crystal AB-stacked bilayer graphene with domain sizes up to hundreds of micrometer was successfully grown on $\mathrm{Cu}$ foils using ethanol as a carbon source. Raman spectroscopy and SAED analysis confirmed the homogeneity and single-crystal nature of the BLG hexagons. By carefully controlling the total pressure of the CVD reaction, the formation of SLG, BLG, and multi-layer areas can be tuned, indicating the dual role of ethanol in graphene growth. This research is beneficial both for the fundamental understanding of the BLG growth mechanism, and the sub-mm AB-stacked BLG is also expected to facilitate the development of functional graphene devices with tunable energy gaps.

\section{Acknowledgment}

We thank Professor Erik Einarsson (SUNY Buffalo) for the helpful discussion. A part of this work was financially supported by Grants-in-Aid for Scientific Research (22226006, 25107002, 15H02219, 15H05760) and IRENA Project by JST-EC DG RTD,

Strategic International Collaborative Research Program, SICORP. We also acknowledge supports from Center for Nano Lithography \& Analysis (The University 
of Tokyo) supported by ‘Nanotechnology Platform’ (project No. 12024046) of MEXT, Japan. A part of this work was also supported by 'Global Center for Excellence for Mechanical Systems Innovation’ (The University of Tokyo), The University of Tokyo, in collaboration with Cadence Corporation. 


\section{References}

[1] Li XS, Cai WW, An JH, Kim S, Nah J, Yang DX, et al. Large-Area Synthesis of High-Quality and Uniform Graphene Films on Copper Foils. Science 2009;324(5932):1312-4.

[2] Bhaviripudi S, Jia XT, Dresselhaus MS, Kong J. Role of Kinetic Factors in Chemical Vapor Deposition Synthesis of Uniform Large Area Graphene Using Copper Catalyst. Nano Lett 2010;10(10):4128-33.

[3] Bae S, Kim H, Lee Y, Xu XF, Park JS, Zheng Y, et al. Roll-to-roll production of 30-inch graphene films for transparent electrodes. Nat Nanotechnol 2010;5(8):574-8.

[4] Vlassiouk I, Regmi M, Fulvio PF, Dai S, Datskos P, Eres G, et al. Role of Hydrogen in Chemical Vapor Deposition Growth of Large Single-Crystal Graphene. Acs Nano 2011;5(7):6069-76.

[5] Zhao P, Kumamoto A, Kim S, Chen X, Hou B, Chiashi S, et al. Self-Limiting Chemical Vapor Deposition Growth of Monolayer Graphene from Ethanol. J Phys Chem C 2013;117(20):10755-63.

[6] Li XS, Magnuson CW, Venugopal A, Tromp RM, Hannon JB, Vogel EM, et al. Large-Area Graphene Single Crystals Grown by Low-Pressure Chemical Vapor Deposition of Methane on Copper. J Am Chem Soc 2011;133(9):2816-9.

[7] Yan Z, Lin J, Peng ZW, Sun ZZ, Zhu Y, Li L, et al. Toward the Synthesis of Wafer-Scale Single-Crystal Graphene on Copper Foils. Acs Nano 2012;6(10):9110-7.

[8] Chen SS, Ji HX, Chou H, Li QY, Li HY, Suk JW, et al. Millimeter-Size Single-Crystal Graphene by Suppressing Evaporative Loss of $\mathrm{Cu}$ During Low Pressure Chemical Vapor Deposition. Adv Mater 2013;25(14):2062-5.

[9] Mohsin A, Liu L, Liu PZ, Deng W, Ivanov IN, Li GL, et al. Synthesis of Millimeter-Size Hexagon-Shaped Graphene Single Crystals on Resolidified Copper. Acs Nano 2013;7(10):8924-31.

[10] Zhou HL, Yu WJ, Liu LX, Cheng R, Chen Y, Huang XQ, et al. Chemical vapour deposition growth of large single crystals of monolayer and bilayer graphene. Nat Commun 2013;4.

[11] Gan L, Luo ZT. Turning off Hydrogen To Realize Seeded Growth of Subcentimeter Single-Crystal Graphene Grains on Copper. Acs Nano 2013;7(10):9480-8.

[12] Hao YF, Bharathi MS, Wang L, Liu YY, Chen H, Nie S, et al. The Role of Surface Oxygen in the Growth of Large Single-Crystal Graphene on Copper. Science 2013;342(6159):720-3.

[13] Yu Q, Jauregui LA, Wu W, Colby R, Tian J, Su Z, et al. Control and characterization of 
individual grains and grain boundaries in graphene grown by chemical vapour deposition. Nat Mater 2011;10(6):443-9.

[14] Huang PY, Ruiz-Vargas CS, van der Zande AM, Whitney WS, Levendorf MP, Kevek JW, et al. Grains and grain boundaries in single-layer graphene atomic patchwork quilts. Nature 2011;469(7330):389.

[15] Chen X, Zhao P, Xiang R, Kim S, Cha J, Chiashi S, et al. Chemical Vapor Deposition Growth of 5 mm Hexagonal Single-Crystal Graphene from Ethanol. Carbon 2015;94.

[16] Zhang YB, Tang TT, Girit C, Hao Z, Martin MC, Zettl A, et al. Direct observation of a widely tunable bandgap in bilayer graphene. Nature 2009;459(7248):820-3.

[17] Mak KF, Lui CH, Shan J, Heinz TF. Observation of an Electric-Field-Induced Band Gap in Bilayer Graphene by Infrared Spectroscopy. Physical Review Letters 2009;102(25).

[18] Yan K, Peng HL, Zhou Y, Li H, Liu ZF. Formation of Bilayer Bernal Graphene: Layer-by-Layer Epitaxy via Chemical Vapor Deposition. Nano Lett 2011;11(3):1106-10.

[19] Liu X, Fu L, Liu N, Gao T, Zhang YF, Liao L, et al. Segregation Growth of Graphene on Cu-Ni Alloy for Precise Layer Control. J Phys Chem C 2011;115(24):11976-82.

[20] Sun ZZ, Raji ARO, Zhu Y, Xiang CS, Yan Z, Kittrel C, et al. Large-Area Bernal-Stacked $\mathrm{Bi}^{-}, \mathrm{Tr}^{-}$, and Tetralayer Graphene. Acs Nano 2012;6(11):9790-6.

[21] Liu LX, Zhou HL, Cheng R, Yu WJ, Liu Y, Chen Y, et al. High-Yield Chemical Vapor Deposition Growth of High-Quality Large-Area AB-Stacked Bilayer Graphene. Acs Nano 2012;6(9):8241-9.

[22] Liu W, Kraemer S, Sarkar D, Li H, Ajayan PM, Banerjeet K. Controllable and Rapid Synthesis of High-Quality and Large-Area Bernal Stacked Bilayer Graphene Using Chemical Vapor Deposition. Chem Mater 2014;26(2):907-15.

[23] Sun HB, Wu J, Han Y, Wang JY, Song FQ, Wan JG. Nonisotherrnal Synthesis of AB-Stacked Bilayer Graphene on Cu Foils by Atmospheric Pressure Chemical Vapor Deposition. J Phys Chem C 2014;118(26):14655-61.

[24] Zhao P, Kim S, Chen X, Einarsson E, Wang M, Song YN, et al. Equilibrium Chemical Vapor Deposition Growth of Bernal-Stacked Bilayer Graphene. Acs Nano 2014;8(11):11631-8.

[25] Kim K, Coh S, Tan LZ, Regan W, Yuk JM, Chatterjee E, et al. Raman Spectroscopy Study of Rotated Double-Layer Graphene: Misorientation-Angle Dependence of Electronic Structure. Phy Rev Lett 2012;108(24). 